University of Nebraska - Lincoln

DigitalCommons@University of Nebraska - Lincoln

2019

\title{
Glycoform Analysis of Alpha1-Acid Glycoprotein by Capillary Electrophoresis Using Electrophoretic Injection
}

Chenhua Zhang

William Clarke

David S. Hage

Follow this and additional works at: https://digitalcommons.unl.edu/chemistryhage

Part of the Medicinal-Pharmaceutical Chemistry Commons

This Article is brought to you for free and open access by the Published Research - Department of Chemistry at DigitalCommons@University of Nebraska - Lincoln. It has been accepted for inclusion in David Hage Publications by an authorized administrator of DigitalCommons@University of Nebraska - Lincoln. 


\title{
Glycoform Analysis of Alpha1-Acid Glycoprotein by Capillary Electrophoresis Using Electrophoretic Injection
}

\author{
Chenhua Zhang, ${ }^{1}$ William Clarke, ${ }^{2}$ and David S. Hage ${ }^{1}$ \\ 1 University of Nebraska-Lincoln \\ 2 Johns Hopkins University School of Medicine
}

\begin{abstract}
Human alpha ${ }_{1}$-acid glycoprotein (AGP) is an acute phase glycoprotein that has a heterogeneous glycosylation pattern. This pattern can change in certain diseases, which has resulted in interest in using AGP glycoforms as potential biomarkers for these diseases. This report describes a method that uses capillary electrophoresis to characterize and analyze AGP glycoforms both in purified samples of AGP and in human serum. This method uses static and dynamic coatings of poly (ethylene oxide) that are applied to a silica capillary for separation of AGP glycoforms in the reversed-polarity mode of CE and in the presence of negligible electroosmotic flow. Electrophoretic injection is performed onto such capillaries by using a stationary stacking interface between the sample and running buffer. In addition, acidic precipitation and desalting are used to allow for the isolation and the analysis of AGP from only $65 \mu \mathrm{L}$ of serum. Up to eleven AGP glycoform bands can be reproducibly separated by this method, with the difference in migration time between neighboring bands being 12 - to almost 60 -fold larger than the standard deviation for the migration time of any given band. A limit of detection down to about $2 \mathrm{nM}$ per glycoform band can be obtained by this method for AGP in serum based on absorbance detection and without the need for further sample modification or labeling.
\end{abstract}

Key words: Alpha, ${ }_{1}$-acid glycoprotein, Capillary electrophoresis, Capillary modification, Electrophoretic injection, Glycoforms, Sample stacking, Serum

Published as Chapter 4 in Terry M. Phillips (ed.), Clinical Applications of Capillary

Electrophoresis: Methods and Protocols, Methods in Molecular Biology, vol. 1972, DOI: https://doi.org/10.1007/978-1-4939-9213-3_4

Copyright (C) 2019 Springer Science+Business Media, LLC, part of Springer Nature. Used by permission. 


\section{Introduction}

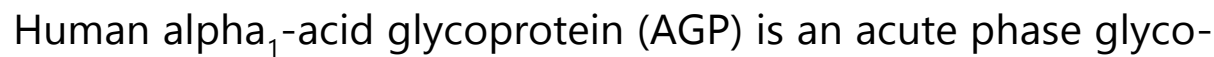
protein that has a single chain of 183 amino acids and two disulfide bonds [1-3]. AGP is a heavily glycosylated protein. The molecular weight of AGP is between 41 and $43 \mathrm{kDa}$, with about $45 \%$ of this mass being due to glycan moieties [1]. As shown in Fig. 1, there are five Nlinked glycosylation sites on AGP, which occur at Asn15, Asn38, Asn54, Asn75, and Asn85. A heterogeneous mixture of bi-, tri-, or tetra-antennary glycans can be attached to these sites [1]. AGP has a low isoelectric point (2.8 to 3.8) due to the presence of multiple sialic acids in these glycan chains $[1,2]$.

Both the concentration and composition of AGP can change with various diseases and clinical conditions [2, 4-7]. For instance, the normal concentration of AGP in serum ranges from 0.5 to $1.0 \mathrm{mg} / \mathrm{mL}$, or 12 to $24 \mu \mathrm{M}$; however, this concentration can increase by up to tenfold in some clinical situations [2]. In addition, alterations in the glycosylation of AGP have been observed. For instance, a decrease in the degree of glycan branching has been reported in patients with infection and systemic lupus erythematosus (SLE) [4]. An increase in $\alpha 1-3$ fucosylation has been noted in pancreatic cancer [5], and an increase in levels of AGP sialylation has been seen in ovarian cancer and lymphoma $[6,7]$. These changes have made AGP glycoforms and their related glycosylation patterns of interest as potential biomarkers for these and other disease states [4-7].

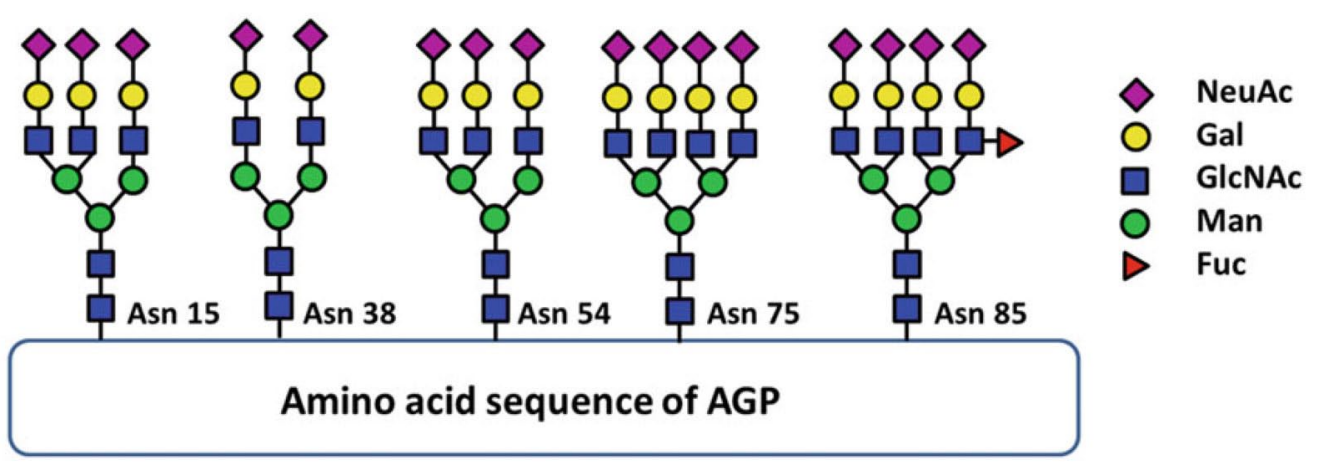

Fig. 1 Examples of complex $\mathrm{N}$-linked glycans that can occur on AGP and the locations of the glycosylation sites on AGP. Abbreviations: GIcNAc N-acetylglucosamine, Man mannose, Gal galactose, Fuc fucose, NeuAc neuraminic acid (or sialic acid). The nomenclature used here for the representation of glycans is based on Ref. [24]. 
Capillary electrophoresis (CE) is one approach that can be used to separate and examine the glycoforms of AGP $[6,8-11]$. During the development of these $C E$ methods, various approaches have been proposed to reduce adsorption of AGP onto the capillary wall and to modify electroomostic flow for improved precision and better resolution of the glycoform bands. These approaches have included the use of buffer additives such as putrescine and urea or the use of capillaries that contained a cross-linked coating of dimethylpolysiloxane $[6,8-10]$. In this report, an alternative method was employed in which static and dynamic coatings of poly (ethylene oxide) (PEO) were applied to a silica capillary. This coating method was used because it has been shown to allow highly reproducible separations of AGP glycoforms and to provide differences in migration times between neighboring glycoform bands that are 12 - to almost 60 -fold larger than the standard deviations of the migration times for these bands [11]. In this report, these coated capillaries were combined with electrophoretic injection and UV absorbance detection to examine the major glycoform bands of human AGP.

The use of absorbance detection alone for AGP tends to provide only modest detection limits in CE due to the short path length of light in the capillary and the capillary's small loading capacity [12]. For instance, a limit of detection of $0.09-0.38 \mu \mathrm{M}$ is obtained for the glycoform bands of AGP when absorbance detection is employed with standard hydrodynamic injection [11]. Mass spectrometry is an alternative detection method that has been used with $C E$, in combination with immunoaffinity extraction, to examine AGP glycoforms in serum; however, the cost of the instrumentation and level of training needed for CE-mass spectrometry has tended to limit its use in routine clinical testing [13-15]. CE with laser-induced fluorescence detection and fluorescent-labeled AGP can also be used to detect AGP glycoforms [16], but this approach adds extra steps and time for sample treatment and modifies the structure of AGP. Electrophoretic injection is a type of electrokinetic injection that can be performed with AGP when there is a negligible amount of electroosmotic flow present in this system [17]. This situation creates an essentially stationary stacking interface between the sample and running buffer during injection. This technique can allow for the sensitive detection of AGP (i.e., down to 0.05-0.2 nM) without the need for AGP derivatization and by using absorbance detection [17]. 
Sample pretreatment was further needed to use CE with absorbance detection and electrophoretic injection for the analysis of AGP in serum (see overall scheme provided in Fig. 2). Multiple chromatographic steps, such as ion-exchange and dye-ligand chromatography, have been used to isolate AGP from serum or plasma; unfortunately, this approach generally requires a relatively large sample volume [18, 19]. Immunoaffinity extraction with anti- AGP immobilized support has been used as well but needs multiple steps for sample loading, elution, and column regeneration [20]. Acidic precipitation has been employed for the pretreatment of AGP from plasma but tends to give a low purity for AGP when compared with immunoaffinity extraction $[20,21]$. The method that is described in this current report combined acidic precipitation, desalting, and electrokinetic injection for the selective enrichment of AGP from serum using sample volumes of as little as $65 \mu \mathrm{L}$ [17].

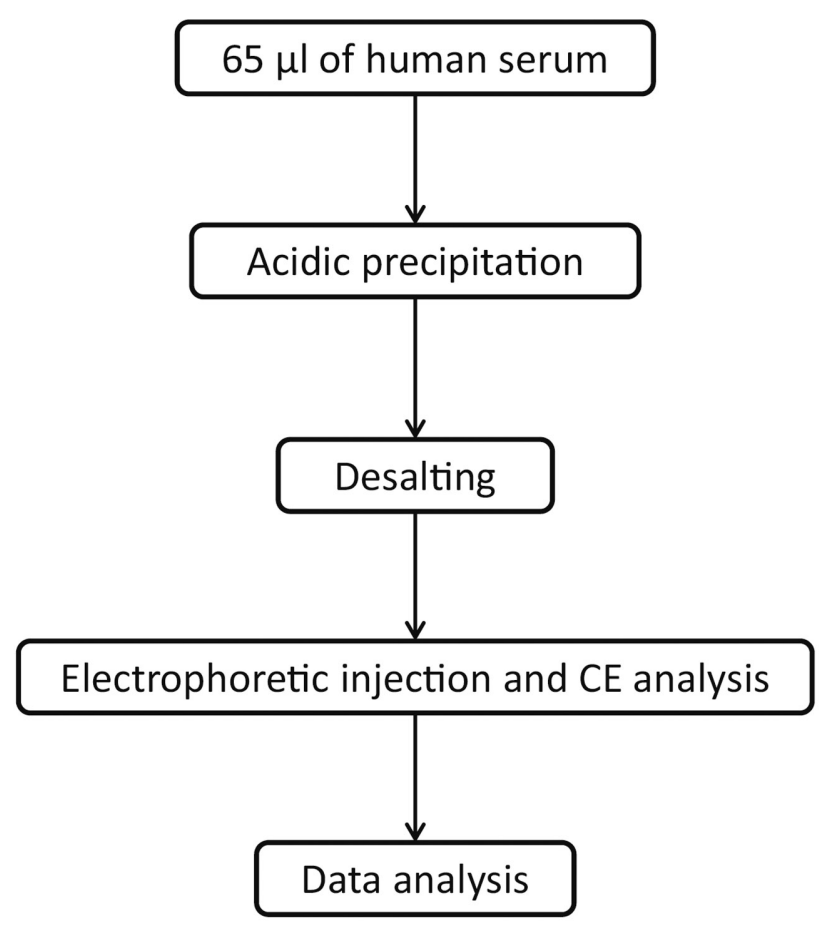

Fig. 2 General scheme for the sample preparation and CE analysis of glycoform bands for AGP that has been isolated from serum. 


\section{Materials}

The water used in the following procedures was prepared with a Milli-Q water purification system (EMD Millipore Corporation); however, other types of water purification systems can also be used.

\subsection{Samples}

1. Commercial samples of normal human AGP ( $\geq 99 \%$ purity, from pooled human plasma) were obtained from Sigma- Aldrich.

2. Commercial samples of human serum were also obtained from Sigma-Aldrich, as were made from pooled human male AB plasma of U.S. origin (sterile-filtered). Each donor that was used to prepare this commercial sample had been tested by ELISA and found to be nonreactive for hepatitis $B$, hepatitis $C$, and human immunodeficiency virus (see Note 1).

3. The specimens from patients with SLE were de-identified and preexisting serum samples that were supplied by W. Clarke; this work has been determined to be exempt from IRB review by the Johns Hopkins School of Medicine, according to the Code of Federal Regulations -45 CFR46.101 b.

\subsection{Equipment}

1. A Beckman P/ACE MDQ capillary electrophoresis system was used in this study. Other brands or models of CE systems with equivalent performance can also be used.

2. Spin column desalting column $(0.5 \mathrm{~mL}, 7 \mathrm{kDa}$ cutoff; Thermo Fisher Scientific).

\subsection{Buffers and Solutions}

1. $0.5 \mathrm{M}$ Perchloric acid solution, $10 \mathrm{~mL}$ : Add $0.429 \mathrm{~mL}$ of perchloric acid (70\%) into a $20 \mathrm{~mL}$ glass vial and add $9.571 \mathrm{~mL}$ water, giving a final perchloric acid concentration of $0.5 \mathrm{M}$ (see Note 2).

2. $1 \mathrm{M}$ Sodium hydroxide $(\mathrm{NaOH})$ solution, $100 \mathrm{ml}$ : Add $4.0 \mathrm{~g}$ of $\mathrm{NaOH}$ into a $150 \mathrm{~mL}$ beaker, add $100 \mathrm{~mL}$ of water and dissolve the $\mathrm{NaOH}$ through stirring or sonication. 
3. $1 \mathrm{M}$ Hydrochloric acid $(\mathrm{HCl})$ solution, $100 \mathrm{~mL}$ : Add $8.333 \mathrm{~mL}$ of $37 \%$ $(\mathrm{w} / \mathrm{w}) \mathrm{HCl}$ into a $100 \mathrm{~mL}$ volumetric glassware and add water to the mark.

4. $0.2 \%(\mathrm{w} / \mathrm{v})$ PEO (viscosity average molecular weight, 8,000,000; Sigma-Aldrich) prepared in $0.1 \mathrm{M} \mathrm{HCl}, 100 \mathrm{~mL}$ : Add $0.2 \mathrm{~g}$ PEO slowly (see Note 3), with stirring, into $100 \mathrm{~mL}$ water that is held in a container within an $80^{\circ} \mathrm{C}$ water bath. Cover the opening of the flask with an upside-down $100 \mathrm{~mL}$ beaker to avoid water evaporation. Stir the solution for $2 \mathrm{~h}$ until all of the PEO has dissolved. After this solution has been cooled to room temperature, add 0.833 $\mathrm{mL}$ of $37 \%$ of $\mathrm{HCl}$ to this solution.

5. $20 \mathrm{mM}$ Acetate buffer, $\mathrm{pH} 4.2$, containing $0.05 \%(\mathrm{w} / \mathrm{v})$ PEO and $0.1 \%$ (w/v) Brij 35, $200 \mathrm{~mL}$ :

Solvent A: 0.05\% (w/v) PEO and 0.1\% (w/v) Brij 35, $300 \mathrm{~mL}$. Add 0.15 $g$ PEO slowly, and with stirring, into a container holding $300 \mathrm{~mL}$ water within an $80^{\circ} \mathrm{C}$ water bath. Cover the opening of the flask with an upside-down $100 \mathrm{~mL}$ beaker to prevent the evaporation of water. Stir this solution for an additional $2 \mathrm{~h}$ until all of the PEO has dissolved. After this solution has been cooled to room temperature, add $0.3 \mathrm{~g}$ Brij 35 to the solution with stirring.

Weigh $0.1196 \mathrm{~g}$ of sodium acetate trihydrate into a $200 \mathrm{~mL}$ beaker. Add approximately $180 \mathrm{~mL}$ of solvent $A$ into this beaker and stir until all the sodium acetate salt has dissolved. Add $178 \mu \mathrm{L}$ of glacial acetic acid to the solution while stirring. Adjust the $\mathrm{pH}$ of this solution to 4.2 by adding small amounts of $1 \mathrm{M} \mathrm{NaOH}$ or $1 \mathrm{M} \mathrm{HCl}$ and while monitoring the $\mathrm{pH}$ of solution (see Note 4). Transfer the final solution into a $200 \mathrm{~mL}$ volumetric glassware and add solvent $A$ to fill this flask to the mark (see Note 5).

\subsection{Spin Desalting Column}

Remove the spin column's bottom closure and loosen the cap without fully removing it. A $2.0 \mathrm{~mL}$ microcentrifuge tube can be used as a collection tube with its cap removed. Place the column in this collection tube and centrifuge at $6600 \mathrm{rpm}(2000 \times \mathrm{g})$ for $1 \mathrm{~min}$ to remove any storage solution that is present in the device. Place a mark on the side of the spin column where the compacted resin is slanted upward; this is to make sure the resin is placed the same way after 
every wash or pre-equilibration step. Place the spin column into a minicentrifuge, with the mark facing outward in all subsequent centrifugation steps. Add $300 \mu \mathrm{L}$ water (i.e., the solution to be used for desalting) to the top of the resin bed in the spin column. Centrifuge at $6600 \mathrm{rpm}$ for $1 \mathrm{~min}$ to remove any water. Repeat the addition of water and this centrifugation process two more times, discarding the water from the collection tube.

\subsection{Fused Silica Capillary}

A fused silica capillary with a length of $72 \mathrm{~cm}$ should be cut from capillary tubing with an inner diameter of $50 \mu \mathrm{m}$ and an outer diameter of $360 \mu \mathrm{m}$, as obtained from Polymicro Technologies (Phoenix, AZ, USA) (see Note 6). This cut can be achieved by using a cleaving stone. Remove the outer polyimide coating from the capillary in the desired region of the detection window. This can be done by placing the capillary on top of the U-shaped opening of an open-ended = in wrench, with this opening being placed at least $11 \mathrm{~cm}$ away from the near end of the capillary. A lighter can then be used to burn off the polyimide coating from the capillary over a length of approximately 5 $\mathrm{mm}$ (see Note 7). This area should then be gently cleaned by using a Kimwipe soaked with ethanol. To install the capillary cartridge for the P/ACE MDQ CE system, insert the long side of the capillary into the outlet side of cartridge. Carefully pull the capillary from the inlet side until the detection window appears in the cartridge window. Select the required aperture clip (size, $100 \times 800 \mu \mathrm{m}$ ) (see Note 8), replace from the back of the cartridge, and use an insertion tool to seat the O-ring into the front of the aperture (see Note 9). Place seal retainer clips on both sides of the capillary and snap these retainer clips into position. Place the cartridge face down against the capillary length template. Use a cleaving stone to cut and remove the marked portion of the capillary. Use the rough side of the cleaving stone to burnish the ends of the capillary until these ends are smooth (see Note 10). After this step, the final capillary will have an effective length of $50 \mathrm{~cm}$ and a total length of $60.2 \mathrm{~cm}$. Mount the cartridge with the prepared fused silica capillary into the CE system. To clean the capillary and activate its interior silanol groups, rinse the capillary with $1 \mathrm{M} \mathrm{NaOH}$ for $30 \mathrm{~min}$ at a pressure of $50 \mathrm{psi}$, and clean the capillary with water for $10 \mathrm{~min}$ at the same pressure $[11,17]$. 


\section{Methods}

\subsection{Acid Precipitation and Desalting}

1. Acid precipitation: Combine a $65 \mu \mathrm{L}$ portion of serum and $130 \mu \mathrm{L}$ of $0.5 \mathrm{M}$ perchloric acid in a $0.6 \mathrm{~mL}$ microcentrifuge tube and vortex mix for $20 \mathrm{~s}$. Place the resulting mixture into a minicentrifuge and allow it to spin at $6600 \mathrm{rpm}$ for $10 \mathrm{~min}$ at room temperature. This step precipitates major proteins from serum while allowing AGP to be recovered in the supernatant [21].

2. Sample desalting: Place a $130 \mu \mathrm{L}$ portion of the supernatant from the acid precipitation step into a spin desalting column. Place the spin desalting column into a $2.0 \mathrm{~mL}$ microcentrifuge tube with the cap removed as a collection tube and spin the column for $2 \mathrm{~min}$ at $6600 \mathrm{rpm}$.

This desalting step helps to improve both the loading capacity and extent of zone broadening in the final CE method through its effect on the relative resistivity (or conductivity) for the sample solution versus the running buffer, as is illustrated in Fig. 3 [17]. The use of both acid precipitation and desalting for sample

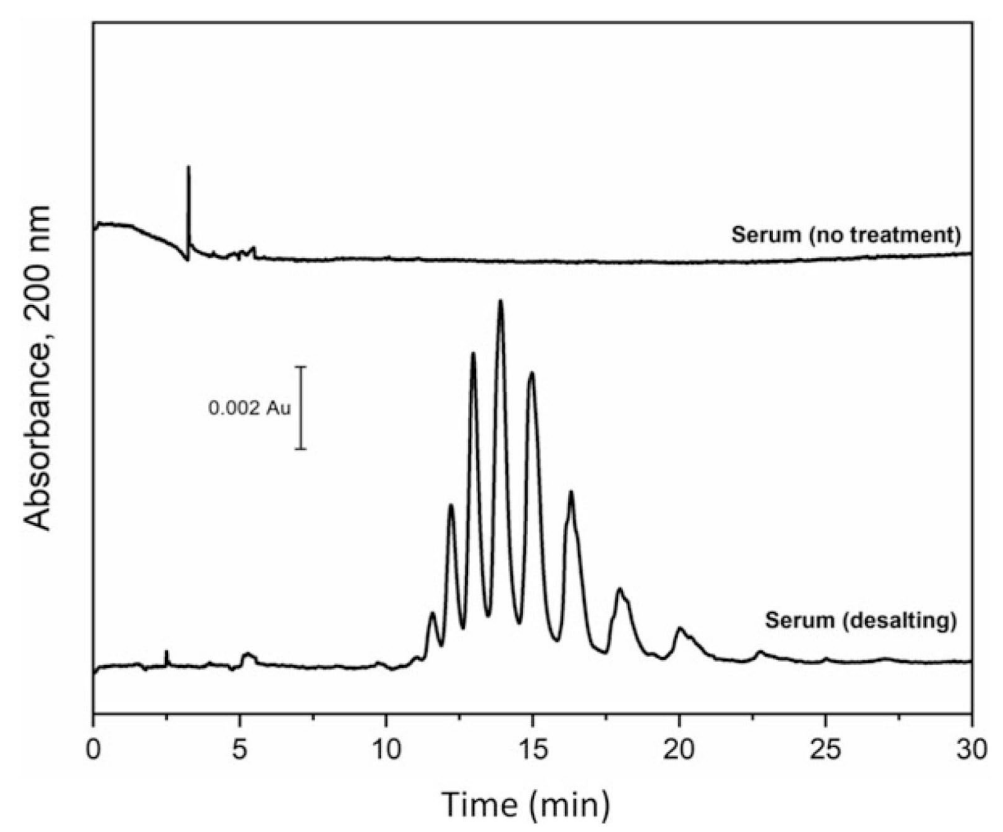

Fig. 3 Electropherograms obtained for AGP glycoforms when using human serum that has been processed by desalting or that was used with no sample pretreatment. This figure is modified with permission from Ref. [17]. 
preparation has also been found to result in good recoveries for AGP glycoforms from serum. For instance, recoveries between 72.3 and $80.9 \%$ have been measured when using this pretreatment method to look at AGP glycoform bands from human serum by CE (see Table 1) [17].

3. Sample dilution: Add the filtrate from the previous step to a $20 \mathrm{ml}$ glass vial that also contains $5 \mathrm{~mL}$ of water (see Note 11). This step is used to reduce the concentration of AGP down to around $1 \mu \mathrm{g} /$ $\mathrm{mL}$ and generates a relatively large volume of a working sample solution that can be employed for multiple injections, or reanalysis as needed.

\subsection{Capillary Electrophoresis and Electrophoretic Injection}

1. CE operating conditions: The following conditions are used for the final method that is described in this report. The capillary temperature should be maintained at $25{ }^{\circ} \mathrm{C}$ during the separation, and the applied voltage for the separation should be set at $-30 \mathrm{kV}$ (see Note 12). AGP glycoforms can be detected by using their absorbance at $200 \mathrm{~nm}[11,17]$.

Table 1 Characteristics of an electrophoretic injection CE method for AGP glycoform analysis in serum using acid precipitation and desalting for sample pretreatment

\begin{tabular}{lrrrr}
$\begin{array}{l}\text { Glycoform } \\
\text { band }\end{array}$ & $\begin{array}{r}\text { Limit of } \\
\text { detection }(n M)^{a}\end{array}$ & $\begin{array}{r}\text { Precision of } \\
\text { migration time }\end{array}$ & $\begin{array}{r}\text { Precision of } \\
\text { peak area }^{b}\end{array}$ & Recoveryc $^{c}$ \\
\hline 3 & 2.5 & $\pm 0.08 \%$ & $\pm 0.86 \%$ & $72.3 \%$ \\
4 & 8.2 & $\pm 0.11 \%$ & $\pm 0.94 \%$ & $80.9 \%$ \\
5 & 11.4 & $\pm 0.12 \%$ & $\pm 0.71 \%$ & $80.4 \%$ \\
6 & 8.5 & $\pm 0.12 \%$ & $\pm 0.52 \%$ & $80.5 \%$ \\
7 & 4.8 & $\pm 0.12 \%$ & $\pm 0.34 \%$ & $80.3 \%$ \\
8 & 2.4 & $\pm 0.13 \%$ & $\pm 0.86 \%$ & $78.2 \%$ \\
9 & 2.1 & $\pm 0.12 \%$ & $\pm 1.18 \%$ & $73.6 \%$ \\
\hline
\end{tabular}

Adapted with permission from Ref. [17]

a. The detection limit was calculated by using a signal-to-noise ratio of three, as based on the standard deviation of the intercept and the slope from a calibration curve for this assay

b. The relative standard deviations listed in these columns are based on measurements made in triplicate $(n=3)$ for normal serum that was spiked with $2.5 \mathrm{~g} / \mathrm{L}$ AGP. The ranges shown represent \pm 1 relative standard deviation

c. The recovery was calculated by dividing the slopes of the corresponding calibration curves that were obtained before and after sample pretreatment with normal serum that had been spiked with a known amount of AGP 
2. Preparation of static and dynamic coatings of PEO: The capillary is first cleaned by using a 5 min rinse with $1 \mathrm{M} \mathrm{NaOH}$, followed by a 3 min rinse with water. The coating is applied by rinsing the capillary for 5 min with $1 \mathrm{M} \mathrm{HCl}$ and then rinsing for $5 \mathrm{~min}$ with a $0.2 \%(\mathrm{w} / \mathrm{v})$ PEO solution that contains $0.1 \mathrm{M} \mathrm{HCl}$. The capillary is then rinsed for 5 min with a running buffer that consists of $\mathrm{pH} 4.2,20 \mathrm{mM}$ acetate buffer containing $0.05 \%(w / v)$ PEO and $0.1 \%(w / v)$ Brij 35. All of these rinse steps should be performed at an applied pressure of 50 psi, and this rinsing procedure should be repeated before each $\mathrm{CE}$ separation. The effects of changing the buffer $\mathrm{pH}$, capillary coating material or concentration of Brij 35 on the separation of AGP glycoforms are discussed under Note 13.

3. Electrophoretic injection: Add $1.2 \mathrm{~mL}$ of the sample to a buffer vial. Carry out electrophoretic injection by using a voltage of $-5 \mathrm{kV}$ that is applied for $5 \mathrm{~min}$ (see Note 14).

This electrophoretic injection step enables selective injection of AGP from a sample that has undergone the pretreatment steps described under Subheading 3.1. The selectivity of this approach is aided by the fact that AGP is one of few proteins that can have a negative charge at an acidic $\mathrm{pH}$ around 4 [22]. The use of an injection time longer than 5 min can result in a higher loading capacity and signal intensity for AGP, as illustrated in Fig. 4a; however, a lower resolution will also be observed under these conditions due
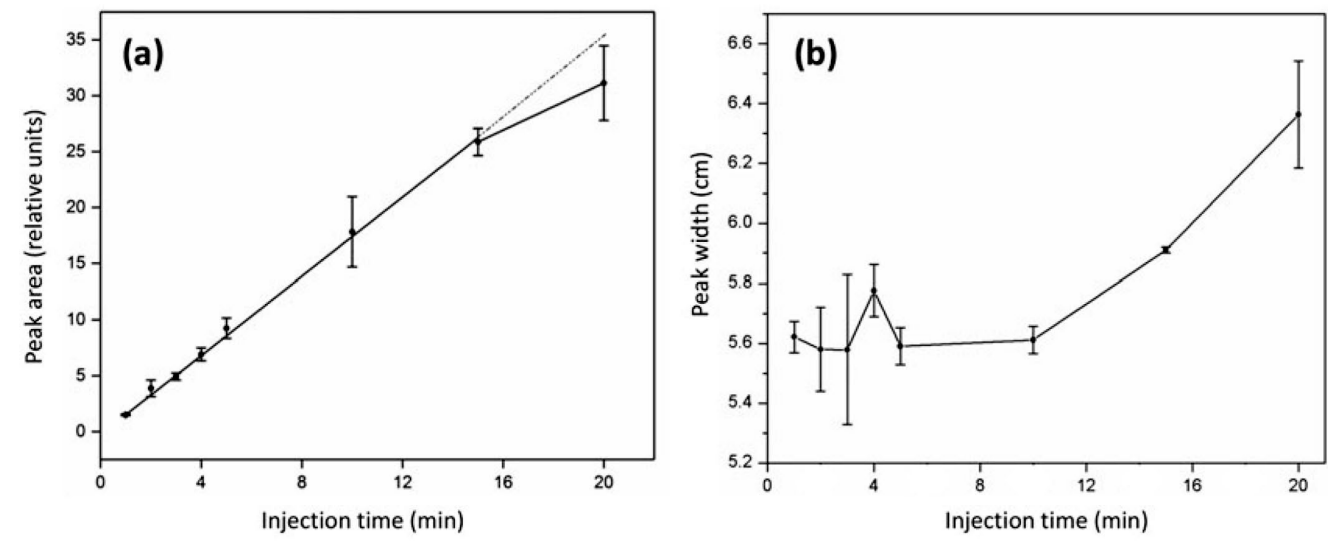

Fig. 4 Relationship between (a) the total peak area for AGP glycoform bands and the time used for electrophoretic injection, and (b) the width for the ninth glycoform band for AGP versus the time used for electrophoretic injection. The error bars represent a range of \pm 1 S.D. $(n=3)$ in (a) and a range of \pm 1 S.D. of the mean $(n=$ 3 ) in (b). The plots used in this figure were adapted with permission from Ref. [17]. 
to the zone broadening that occurs during injection, as shown in Fig. 4b. In contrast, the use of an injection time that is shorter than 5 min will result in smaller signal intensity with similar broadening to what is seen at an injection time of $5 \mathrm{~min}$. Based on these trends, an injection time of 5 min has been determined to provide a good compromise between detection limits, overall analysis times, and peak resolution [17].

4. Capillary cleaning and storage: After a single separation or multiple separations that are carried out as a sequence, the capillary should be rinsed with $1 \mathrm{M} \mathrm{NaOH}$ at 50 psi for $15 \mathrm{~min}$ and then rinsed with water at 50 psi for 10 min. The capillary should be stored in water when it is not in use (see Note 15).

\subsection{Data Analysis}

Most of the following procedures for data analysis are specific for the type of CE system and analysis software that were used in this study. Equivalent procedures can be employed with alternative CE systems or software.

1. An electropherogram that has been obtained by the CE system can be exported as a .cdf file for analysis of its peaks by a program such as Peakfit version 4.12.

2. Use the "section" option to select a specific region of the electropherogram for closer analysis (i.e., a suggested range of 10-25 min for most applications of this CE method).

3. To carry out a baseline correction using Peakfit, select the "progressive line" option. This option can be used to correct for artifacts such as baseline drifting.

4. When using Peakfit for data analysis, select the options "Chromatography" and "Exponentially modified Gaussian or EMG" for peak type. Select the options "Vary widths," "Vary shape," and "Allow negative" for auto scan. Click on the electropherogram to add multiple simulated peaks and adjust their shape to give an approximate fit to the electropherogram.

5. When using Peakfit, select and click the button that says "Fast peak fit with numerical update" until the best fit is obtained. Next, select 
and click the options "Review fit" and "Numeric" to acquire the measured parameters for each individual peak or band. To obtain more parameters for the simulated peaks, select "Options" to add in additional factors such as "Chromatography analysis."

\subsection{Analysis of AGP Glycoform Bands in Serum Samples}

1. The electropherograms in Fig. 5a show some typical results that were obtained by the overall sample pretreatment and CE method for normal and SLE serum samples. The migration times and peak areas of the individual glycoform bands in these electropherograms were obtained by following the data analysis procedures that were described under Subheading 3.3.

2. The total peak area for AGP in the electropherogram can be obtained in this particular method by using a summation of the peak areas for the individual glycoform bands (see Note 16). A correction of the peak areas based on their apparent mobilities, as is common in other CE methods, is not needed in this approach with negligible electroosmosis because the bias of peak area in detection due to differential mobility has already been corrected during the electrophoretic injection process [17]. The relative peak area due to each
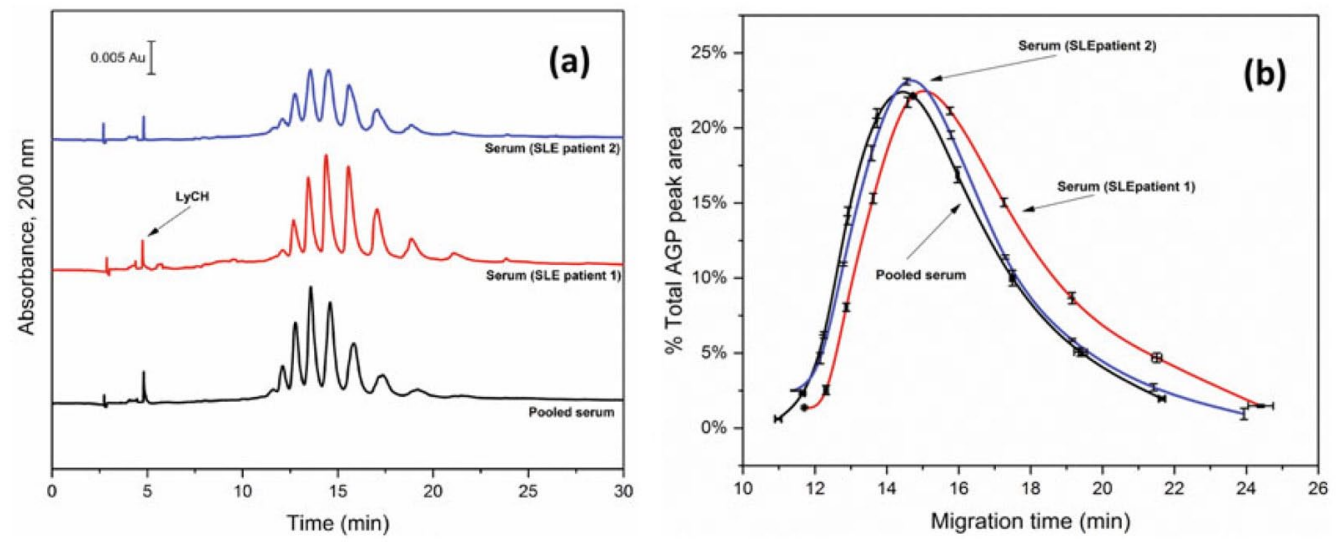

Fig. 5 (a) Electropherograms obtained for AGP from SLE serum or pooled normal serum, in which the mobility marker (Lucifer yellow $\mathrm{CH}$, $\mathrm{LyCH}$ ) was introduced by hydrodynamic injection after sample injection. (b) Glycoform patterns obtained for AGP from SLE serum or pooled normal serum by this CE method; the error bars shown for the migration times and \% peak areas represent \pm 1 S.D. of the mean ( $n$ = 3). These plots are reproduced with permission from Ref. [17]. 
individual band can be found by dividing the separate band/peak areas by the total peak band/peak for AGP.

3. The glycoform band distribution pattern for AGP can be visualized by making a plot of the percentage peak area for each glycoform band (placed on the $y$-axis) versus the migration time of each individual band (on the $x$-axis) [17]. Examples of such plots are shown in Fig. 5b.

4. This sample preparation and CE method has been found to have a limit of detection ranging from 2.1 to $11.3 \mathrm{nM}$ for the major glycoform bands of AGP in human serum [17]. These limits of detection are approximately 3000 -fold lower than the concentration of AGP that is normally observed in human serum and allow this method to be used with only $65 \mu \mathrm{l}$ of serum without the need for any sample labeling [17].

5. The precision of the migration times for the AGP glycoform bands is between \pm 0.08 and $0.13 \%$; the precisions for the peak areas are between \pm 0.34 and $1.18 \%$ when using an internal standard to correct the peak areas for their variations in loading capacity during the injection process (see Table 1). This reproducible separation allows the direct use of migration times to identify and distinguish between AGP glycoform bands in this method [17].

\section{Notes}

1. Any work with human samples such as serum that may contain bloodborne pathogens should be handled according to appropriate biosafety protocols and in BSL-2 facilities.

2. Use appropriate chemical hazard precautions and facilities when handling perchloric acid [23].

3. This step is to avoid the formation of aggregates of PEO, which will be difficult to dissolve.

4. Be sure to calibrate the $\mathrm{pH}$ meter before use, as the separation of AGP glycoforms by $\mathrm{CE}$ can be sensitive to a small change in the $\mathrm{pH}$ of the running buffer [11].

5. Do not filter this buffer solution because the PEO may not be able to pass through the filter (e.g., one using a $0.2 \mu \mathrm{m}$ pore size membrane).

6. The protocol described in this report has been developed for these particular capillary dimensions. The use of other capillary dimensions will 
affect the resolution, overall analysis time, and peak broadening that are obtained in the final method, and will require further optimization of parameters such as the applied voltage and capillary pretreatment/rinsing protocol, among other factors.

7. Extra care needs to be taken when handling a capillary that contains a region without a polyimide coating because the exposed region of this capillary can be quite brittle.

8. Make sure the O-ring has been removed from the aperture clip before installing the aperture clip. A preinstalled O-ring inside the aperture clip can crack the capillary when you are installing the aperture clip.

9. Make sure the O-ring lays flat after its installation. An improper orientation of the O-ring will block the light path and create resistance when connecting the optical fiber with the aperture clip.

10. A magnifying lens can be used to inspect the end of capillary. A rough end on the capillary will result in a tailing effect for all the AGP glycoform bands in the final method and should be avoided.

11. The remaining contents of the serum sample are diluted by 115.4 -fold as a result of the overall pretreatment procedure.

12. An applied voltage of $-30 \mathrm{kV}$ is the highest setting that is available in the CE system that was used in this work. A lower applied voltage can be used but will generate a smaller electric field, leading to reduced migration speeds and longer analysis times. The peaks or bands will also appear broader in the time domain under lower voltage conditions.

13. Several other $\mathrm{pH}$ conditions have been evaluated for use with this method. When using a $\mathrm{pH}$ range of 3.8 to 5.0 for the running buffer, it was found that the best compromise between resolution and peak broadening was obtained at $\mathrm{pH} 4.2$ [11]. As is shown in Fig. 6, an increase in resolution was observed for AGP glycoform bands in going from $\mathrm{pH} 5.0$ to 4.2; however, the zone broadening increased and led to a reduction in resolution when going from $\mathrm{pH} 4.2$ to 3.8 .

The effect of changing the concentration of Brij 35 as a buffer additive has been evaluated over the range of 0 to $0.5 \%(\mathrm{w} / \mathrm{v})$. It was found that the use of $0.1 \%$ Brij 35 gave the best resolution for the glycoform bands, as is illustrated in Fig. 7 [11].

Table 2 compares the results that were obtained when using various types of coatings for the CE capillary. It was found that the use of PEO as both a static and dynamic coating reagent resulted in the greatest reduction of electroosmotic flow and the shortest overall analysis time when compared to capillary coatings based on poly(vinyl alcohol) (or PVA) or dextran for the separation of AGP glycoforms [11]. 


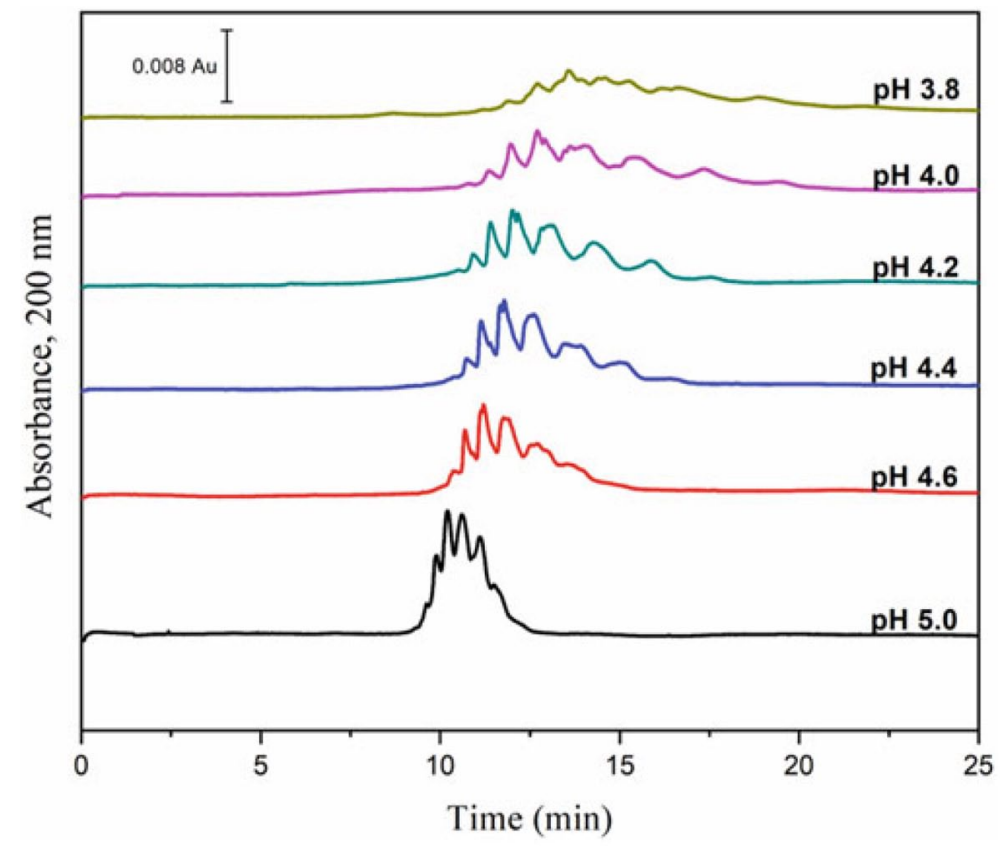

Fig. 6 Electropherograms showing the effect of $\mathrm{pH}$ on the separation and resolution of AGP glycoform bands by CE. This figure is reproduced with permission from Ref. [11].

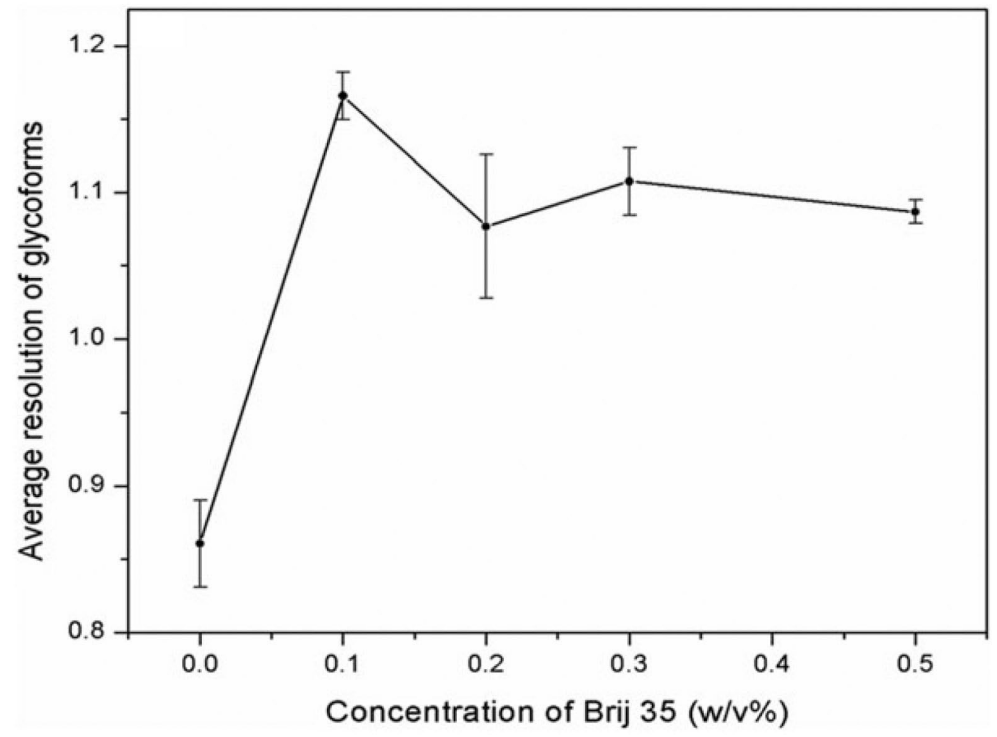

Fig. 7 Average resolution obtained for AGP glycoform bands by CE as a function of the concentration of Brij 35 that was placed into the running buffer. This figure was reproduced with permission from Ref. [11]. The error bars represent a range of \pm 1 S.D. $(n=3)$. 
Table 2 Effect on electroosmotic flow when using various modification agents and methods to treat fused silica capillaries ${ }^{a}$

\begin{tabular}{lrr} 
Modification agent and method & $\begin{array}{r}\text { Mobility due to } \\
\text { electroosmotic flow, } \\
\left(\times 10^{-3} \mathrm{~cm}^{2} \mathrm{~min}^{-1} \mathrm{~V}^{-1}\right)\end{array}$ & $\begin{array}{r}\text { Reduction in } \\
\text { electroosmotic flow } \\
(\%)\end{array}$ \\
\hline Poly(vinyl alcohol) (or PVA) - Permanent coating & $7.05( \pm 0.02)$ & $65.8( \pm 1.4)$ \\
PVA - Permanent plus dynamic coating & $7.09( \pm 0.23)$ & $66.8( \pm 1.8)$ \\
PVA - Static coating & $11.04( \pm 0.27)$ & $48.0( \pm 2.6)$ \\
PVA - Static plus dynamic coating & $9.85( \pm 0.06)$ & $52.3( \pm 2.0)$ \\
Poly (ethylene oxide) (or PEO) - Static coating & $1.83( \pm 0.01)$ & $91.1( \pm 0.4)$ \\
PEO - Static plus dynamic coating & $0.222( \pm 0.002)$ & $98.9( \pm 0.1)$ \\
Dextran - Static coating & $9.76( \pm 0.01)$ & $52.8( \pm 2.0)$ \\
Dextran - Static plus dynamic coating & $9.89( \pm 0.10)$ & $51.7( \pm 2.1)$ \\
\hline
\end{tabular}

Reproduced with permission from Ref. [11]

a. The mobility due to electroosmotic flow for a fused silica capillary was $20.66( \pm 0.87) \times$ $10^{-3} \mathrm{~cm}^{2} \mathrm{~min}^{-1} \mathrm{~V}^{-1}$. The electroosmotic flow was measured in $\mathrm{pH} 4.2,20 \mathrm{mM}$ acetate buffer with a sample containing $1.0 \mathrm{~g} / \mathrm{L}$ thiourea in water. All of the results represent the average of three trials, where the number in parentheses corresponds to a range of $\pm 1 \mathrm{~S}$.D

14. The "inject" event during the method program has a time limit of up to $99 \mathrm{~s}$. For the particular CE system that was employed in this study, a "separate" event can be used instead for sample injection during the method program for applying a voltage for $5 \mathrm{~min}$. The sample can be added to a $2.0 \mathrm{~mL}$ vial and placed into the buffer tray to use this event.

Every sample vial is only used for injection once in this approach because electrophoretic injection will deplete AGP from the sample vial without depleting the buffer or solvent. This means the concentration of AGP in the original sample vial will be reduced after even a single injection step.

15. This procedure should be performed right away after a separation sequence or multiple separations to avoid the permanent adsorption of serum proteins to the inner surface of the capillary, as may occur if this capillary has been left unrinsed for a long time.

16. The peak area obtained by this method represents the true value without bias due to differences in the mobilities of the separate AGP glycoform bands [17]. When two analytes with the same quantity are injected by this approach, the analyte peak that has the longer migration time will have a larger area than the peak for the second analyte with the shorter migration time. This bias effect is automatically corrected during electrophoretic injection because a smaller amount is injected for an analyte with a smaller apparent mobility. More details on the theory of this approach can be found in Ref. [17]. 
Acknowledgment - This work was supported by the National Institutes of Health under grant R01 GM044931.

\section{References}

1. Fournier T, Medjoubi N, Porquet D (2000) Alpha-1-acid glycoprotein. Biochim Biophys Acta 1482:157-171

2. Ceciliani F, Pocacqua V (2007) The acute phase protein $\alpha_{1}$-acid glycoprotein: a model for altered glycosylation during diseases. Curr Protein Pept Sci 8:91-108

3. Schönfeld DL, Ravelli RBG, Mueller U, Skerra A (2008) The 1.8- $\AA$ crystal structure of $\alpha_{1}$-acid glycoprotein (orosomucoid) solved by UV RIP reveals the broad drug-binding activity of this human plasma lipocalin. J Mol Biol 384:393-405

4. Mackiewicz A, Marcinkowska-Pieta R, Ballou S, Mackiewicz S, Kushner I (1987) Microheterogeneity of alpha 1-acid glycoprotein in the detection of intercurrent infection in systemic lupus erythematosus. Arthritis Rheum 30:513-518

5. Balmaña M, Giménez E, Puerta A, Llop E, Figueras J, Fort E, Sanz-Nebot V, de Bolo's C, Rizzi A, Barrabés S, de Frutos M, Peracaula R (2016) Increased $\alpha 1-3$ fucosylation of $\alpha$-1-acid glycoprotein (AGP) in pancreatic cancer. J Proteome 132:144-154

6. Lacunza I, Sanz J, Diez-Masa JC, de Frutos M (2006) CZE of human alpha-1-acid glycoprotein for qualitative and quantitative comparison of samples from different pathological conditions. Electrophoresis 27:4205-4214

7. Lacunza I, Sanz J, Diez-Masa JC, de Frutos M (2007) Erratum: CZE of human alpha-1-acid glycoprotein for qualitative and quantitative comparison of samples from different pathological conditions. Electrophoresis 28:492

8. Pacáková V, Hubená S, Tichá M, Maděra M, Štulík K (2001) Effects of electrolyte modification and capillary coating on separation of glycoprotein isoforms by capillary electrophoresis. Electrophoresis 22:459-463

9. Kinoshita M, Murakami E, Oda Y, Funakubo T, Kawakami D, Kakehi K, Kawasaki N, Morimoto K, Hayakawa T (2000) Comparative studies on the analysis of glycosylation heterogeneity of sialic acidcontaining glycoproteins using capillary electrophoresis. J Chromatogr A 866:261-271

10. Kakehi K, Kinoshita M, Kawakami D, Tanaka J, Sei K, Endo K, Oda Y, Iwaki M, Masuko T (2001) Capillary electrophoresis of sialic acidcontaining glycoprotein. effect of the heterogeneity of carbohydrate chains on glycoform separation using an $\alpha_{1}$-acid glycoprotein as a model. Anal Chem 73:2640-2647

11. Zhang C, Hage DS (2016) Glycoform analysis of alpha ${ }_{1}$-acid glycoprotein by capillary electrophoresis. J Chromatogr A 1475:102-109

12. Simpson SL, Quirino JP, Terabe S (2008) On-line sample preconcentration in capillary electrophoresis. J Chromatogr A 1184:504-541 
13. Ongay S, Martín-Á Ivarez PJ, Neusüß C, de Frutos M (2010) Statistical evaluation of CZE-UV and CZE-ESI-MS data of intact $\alpha$-1-acid glycoprotein isoforms for their use as potential biomarkers in bladder cancer. Electrophoresis 31:3314-3325

14. Ongay S, Neusüß C, Vaas S, Díez-Masa JC, de Frutos M (2010) Evaluation of the effect of the immunopurification-based procedures on the CZE-UV and CZE-ESI-TOF-MS determination of isoforms of intact alpha-1-acid glycoprotein from human serum. Electrophoresis 31:1796-1804

15. Marino K, Bones J, Kattla JJ, Rudd PM (2010) A systematic approach to protein glycosylation analysis: a path through the maze. Nat Chem Biol 6:713-723

16. Garrido-Medina R, Puerta A, Rivera-Monroy Z, de Frutos M, Guttman A, DíezMasa JC (2012) Analysis of alpha-1-acid glycoprotein isoforms using CE-LIF with fluorescent thiol derivatization. Electrophoresis 33:1113-1119

17. Zhang C, Bi C, Clarke W, Hage DS (2017) Glycoform analysis of alpha ${ }_{1}$-acid glycoprotein based on capillary electrophoresis and electrophoretic injection. J Chromatogr A 1523:114-122

18. Kishino S, Miyazaki K (1997) Separation methods for glycoprotein analysis and preparation. J Chromatogr B 699:371-381

19. Kremmer T, Szöllösi É, Boldizsár M, Vincze B, Ludányi K, Imre T, Schlosser G, Vékey K (2004) Liquid chromatographic and mass spectrometric analysis of human serum acid alpha-1-glycoprotein. Biomed Chromatogr 18:323-329

20. Ongay S, Lacunza I, Díez-Masa JC, Sanz J, de Frutos M (2010) Development of a fast and simple immunochromatographic method to purify alpha 1-acid glycoprotein from serum for analysis of its isoforms by capillary electrophoresis. Anal Chim Acta 663:206-212

21. Stumpe M, Miller C, Morton NS, Bell G, Watson DG (2006) High-performance liquid chromatography determination of alpha ${ }_{1}$-acid glycoprotein in small volumes of plasma from neonates. J Chromatogr B 831:81-84

22. Righetti PG, Caravaggio T (1976) Isoelectric points and molecular weights of proteins. J Chromatogr A 127:1-28

23. Muse LA (1972) Safe handling of the perchloric acid in the laboratory. J Chem Educ 49: A463

24. Varki A, Cummings RD, Esko JD, Freeze HH, Stanley P, Marth JD, Bertozzi CR, Hart GW, Etzler ME (2009) Symbol nomenclature for glycan representation. Proteomics 9:5398-5399 